\title{
Psicooncología
}

ISSN: 1696-7240

\section{Estructura básica de una página web para apoyo psicológico a adoles- centes cuyos padres tienen cáncer}

\author{
Jacqueline Hellmüller ${ }^{1}$, Tomás Blasco Blasco ${ }^{2 *}$
}

Recibido el 3 de mayo de 2018 / Aceptado el 20 de diciembre de 2018

Resumen. Cuando un padre o una madre enferma de cáncer, a los hijos les afecta el diagnóstico, siendo los adolescentes el grupo más vulnerable a sufrir las consecuencias. Éstos consideran que sus necesidades son poco escuchadas. Por lo tanto se propone crear los elementos de una página web de apoyo psicológico para adolescentes con padres con cáncer. A partir de entrevistas semiestructuradas con adolescentes, padres afectados, y psicooncólogas, y de la comparación de estos datos con los provenientes de la literatura, hemos llegado a la conclusión de que la página web debería incluir tres elementos fundamentales: información, asesoramiento para el uso de estrategias de afrontamiento, y posibilidad de interactuar con otros adolescentes en la misma situación y también con profesionales. Es también muy necesario que la página web no sea iatrogénica por lo que se propone un sistema de filtros que permitan dirigir al adolescente a los contenidos adecuados para su situación, evitando, en la medida de lo posible, que realice interpretaciones y desarrolle expectativas erróneas que puedan generarle confusión o malestar.

Palabras claves: cáncer parental, Adolescentes, apoyo online, funcionamiento psicosocial.

\section{[en] Basic structure of a web page for psychological support to adolescents whose parents have cancer}

\begin{abstract}
When a father or a mother gets diagnosed with cancer, children are affected too. Adolescents are the most vulnerable group to suffer the consequences. They consider that their surrounding do not listen to their necessities. That is why we propose an innovative approach to create the elements of a psychological support website for adolescents with parental cancer. The procedure was based on conducting semistructured interviews with adolescents, affected parents and psycho-oncologist. Results been given from the comparison between the interviews that have been done in this research and the results of the scientific literature. We concluded that the website should include three fundamental elements: Information, coping strategies counseling, and the possibility to interact with other adolescents in the same situation as well as with professionals. It is of great importance to avoid that the website should be iatrogenic. That is the reason why we propose a filter's system that allows to direct the adolescent to the adequate content for his/her situation. These filters avoid, as much as possible, the wrong misinterpretation and expectations that can generate confusion and discomfort.

Keywords: parental cancer, adolescents, online support, psychosocial functioning.
\end{abstract}

1 Unitat de Psicologia Bàsica. Universitat Autònoma de Barcelona.

E-mail: jacqueline.hellmueller@gmail.com

2 Unitat de Psicologia Bàsica. Universitat Autònoma de Barcelona.

E-mail: tomas.blasco@uab.es

* Dirección de correspondencia: Tomás Blasco. Unidad de Psicología Básica. Edifici B. Universitat Autònoma de Barcelona. 08193 Bellaterra (Barcelona). E-mail: tomas.blasco@uab.es 
Sumario. 1. Introducción 2. Objetivos de la propuesta 3. Métodos utilizados para determinar los elementos de la página web 3.1. Análisis de las entrevistas 3.2. Comparación entre entrevistas y literatura e implicaciones para la página web 4. Propuesta para la elaboración de los elementos de la página web 5. Limitaciones 6 . Conclusiones 7. Referencias bibliográficas.

Cómo citar: Hellmüller J, Blasco Blasco T. Estructura básica de una página web para apoyo psicológico a adolescentes cuyos padres tienen cáncer. Psicooncología 2019; 16(1): 191-204. doi: 10.5209/ PSIC.63655.

\section{Introducción}

El cáncer es la segunda causa de muerte en todo el mundo. Solo en España en el año 2015 se diagnosticaron 247.771 nuevos casos $^{(1)}$. Muchos de los pacientes con cáncer tienen familia e hijos. Hay que tener en cuenta que, cuando aparece el diagnóstico de cáncer con la carga psicológica que conlleva, no sufre solo el paciente sino toda la familia y especialmente los hijos ${ }^{(2)}$. Se ha visto que los hijos de un padre o una madre con cáncer tienen un mayor riesgo de sufrir problemas emocionales, conductuales, sociales, físicos y cognitivos así como problemas de concentración en la escuela ${ }^{(2-4)}$. Les cuesta manejar la incertidumbre de los resultados de las pruebas médicas y les preocupa que el cáncer pueda ser hereditario ${ }^{(5)}$. Se asustan al ver la debilidad física o los cambios físicos y temen perder a su madre o padre enfermo ${ }^{(3,4,6)}$. Los hijos a veces expresan estos miedos y preocupaciones mediante síntomas somáticos ${ }^{(6)}$.

Por el momento vital en el que se encuentran, los adolescentes son un grupo especialmente vulnerable a sufrir las consecuencias del cáncer parental. Una posible explicación se encuentra en el mayor desarrollo de sus habilidades cognitivas, pues tienen mayores capacidades para pensar de manera analítica, lógica y reflexiva y toman más en consideración la perspectiva de otras personas. Todo eso puede hacer que los adolescentes reconozcan mejor la gravedad de la situación y las consecuencias de la enfermedad ${ }^{(7,8)}$. Además los hijos mayores están más preocupados por el bienestar del padre o de la madre enfermo que por su propio bienestar y acaban siendo cuidadores informales ${ }^{(2)}$. El cáncer cambia las rutinas diarias de los adolescentes e incluso puede haber un cambio de roles con los padres, teniendo que asumir ellos más responsabilidad en casa o en el cuidado de los hermanos. Esto puede provocar una reducción del tiempo que pasan con sus amigos y su vida social puede verse interrumpida. Como respuesta a la enfermedad parental, algunos hijos se terminan aislando ${ }^{(4)}$. Por lo tanto, los adolescentes necesitan apoyo para poder afrontar la enfermedad parental, una necesidad que en la mayoría de los casos no está suficientemente cubierta. Niemelä et al. ${ }^{(9)}$ concluyen que los hijos son el grupo olvidado cuando los padres afrontan esta enfermedad, a pesar de la alta necesidad de soporte y apoyo que tienen. Las intervenciones psicosociales se centran muchas veces en el padre o la madre enfermo y se olvidan de los hijos. Por otra parte, el momento vital por el que están pasando los adolescentes y la mayor independencia de los padres, hace que estos se den menos cuenta del estado psicológico en el que se encuentra su hijo/a. Hay estudios que indican que los padres subestiman los problemas de sus hijos, minimizando su percepción respecto a la de los adolescentes y subestimando el impacto emocional que tiene su diagnóstico en el/la hijo/a ${ }^{(2,3,8,10)}$. Según Fernández Sánchez et al. ${ }^{(11)}$ las intervenciones en familiares están más solicitadas en la fase del tratamiento activo y en la fase del 
duelo. Consultan por malestar emocional, problemas relacionados con el duelo, pero también por la necesidad de orientación. No obstante, hay que tener en cuenta que, según Küçükoğlu y Çelebioğlu ${ }^{(7)}$ el momento justo después del diagnóstico también es un momento muy crítico para los adolescentes.

En relación a las necesidades de los adolescentes en esta situación, Huang et al. ${ }^{(4)}$ concluyen que ellos perciben que en general el entorno presta poca atención a sus necesidades. Experimentan una falta de soporte y no sienten que el entorno les entienda cuando hablan de sus sentimientos o experiencias, por lo tanto perciben que sus sentimientos son ignorados por otros. Expresan una intensa necesidad de recibir más información y piensan que les hubiera ayudado que el personal sanitario les recomendara una página web sobre cáncer parental ${ }^{(3)}$. Quieren conocer la definición de cáncer, sus causas, las opciones de tratamiento, los posibles efectos secundarios que este tratamiento puede tener, el pronóstico, y el riesgo para ellos de heredarlo. Solo en los estudios de Sears y Sheppard ${ }^{(12)}$ y Morris et al. ${ }^{(2)}$ se menciona la importancia que tiene para los hijos el poder implicarse en el proceso de la enfermedad.

En referencia a los recursos que a los hijos ayudan a sobrellevar mejor la situación, los estudios señalan que los adolescentes aprecian el contacto con los profesionales de salud y el recibir información de ellos sobre cómo pueden ser las reacciones psicológicas normales y comunes en esta situación ${ }^{(3,4)}$. Necesitan un espacio donde ellos y no el cáncer parental sea el foco de atención ${ }^{(13)}$. Asimismo, está demostrado que los adolescentes encuentran útil poder hablar con otros adolescentes que están en, o han pasado por, la misma experiencia, ya que muchas veces tienen la sensación de que si alguien no ha pasado por la misma situación no puede entenderles. Poder interactuar con otros adolescentes que están en la misma situación les ayuda a sentirse menos solos e incrementa la sensación de soporte ${ }^{(4,13)}$, ya que tienen un fuerte deseo de compartir su experiencia con alguien, pero les es difícil encontrar una persona para hablar ${ }^{(4)}$, ya que por miedo a que los hijos sufran, los padres puedan prescindir de hablar del cáncer ${ }^{(14)}$. A pesar de esto, una comunicación abierta en la familia y el poder expresar libremente los sentimientos, se asocia con menos ansiedad y depresión, con una mayor capacidad de resolución de problemas y, en general, con una mejor adaptación por parte de los adolescentes ${ }^{(15,16)}$.

En resumen, podemos decir que para poder afrontar la situación de cáncer parental los adolescentes utilizan diversas estrategias, entre ellas la distracción para mantener la mayor normalidad posible en su vida. También buscan apoyo social, ayudan al padre enfermo y buscan darle un sentido a la enfermedad. En base a esto, los profesionales sanitarios deben facilitar el afrontamiento de los adolescentes mediante estrategias que han demostrado una mayor eficacia, como las estrategias centradas en el problema o las emociones y no las estrategias basadas en la evitación ${ }^{(2,10,17)}$.

En este contexto, y teniendo en cuenta que los adolescentes han integrado el uso de internet en su vida diaria, cabe la posibilidad de que éste pueda erigirse como una herramienta efectiva para apoyarles en el afrontamiento del cáncer parental ${ }^{(4)}$. Los adolescentes no solo usan internet para buscar información sobre qué es el cáncer y su tratamiento, sino también para expresar emociones, sentimientos, sus experiencias con el cáncer y buscar consejos para desenvolverse mejor en la situación ${ }^{(5)}$. Internet les sirve para poder comunicarse con otros y no sentirse $\operatorname{solos}^{(18)}$. La gran ventaja de internet es que es accesible durante las 24 horas y no hay barreras geográficas a la vez que proporciona un contacto confidencial y anónimo ${ }^{(19,20)}$. También puede beneficiar a adolescentes que por miedo a preocupar sus padres no externalizan sus respuestas 
emocionales, ya que en la adolescencia es crucial tener una respuesta a las necesidades emocionales en situaciones difíciles. Una página web les puede ayudar a normalizar la experiencia y conectarles con otros que están pasando por la misma situación. Para las familias el buscar apoyo en internet puede erigirse como un factor clave en el afrontamiento del cáncer parental. Y para familias que viven por ejemplo en una zona rural, internet puede ser su única fuente de apoyo ${ }^{(20-22)}$. A pesar de ello comunicarse por medio de la tecnología no puede sustituir el contacto de cara a cara con un profesional sanitario, pero puede acercar a los adolescentes a los servicios sanitarios ${ }^{(19)}$.

\section{Objetivos de la propuesta}

Teniendo en cuenta la idoneidad de un apoyo tecnológico para adolescentes con padres con cáncer, el objetivo general para este trabajo es elaborar una relación de los contenidos básicos que debe tener una página web de apoyo psicológico para adolescentes con padres con cáncer que facilite a los adolescentes, la adaptación a esta situación. Teniendo en cuenta la clasificación de la World Health Organization, que habla de tres fases de la adolescencia: adolescencia temprana (10-13 años), adolescencia media (14 a 16 años) y adolescencia tardía (17 a 19 años), la página web se diseñará para adolescentes con edades entre 10 a 19 años que viven en España y cuyo padre o madre sufre cáncer o ha sufrido cáncer.

En este trabajo pretendemos identificar qué elementos debería tener una página web para:

1. Proporcionar a los adolescentes la información que necesiten sobre el cáncer.

2. Dar a conocer posibles pautas y estrategias para afrontar la situación.

3. Ofrecer contacto con adolescentes que están pasando por la misma situación.

4. Acercar los adolescentes a profesionales sanitarios que les ayudan a manejar la situación.

5. Evitar efectos iatrogénicos.

\section{Métodos utilizados para determinar los elementos de la página web}

Adicionalmente a la revisión de la literatura encontrada, realizamos un total de 10 entrevistas semiestructuradas (tres a adolescentes cuyos padres tienen o han tenido cáncer, cinco a madres o padres pacientes de cáncer, y dos a psicooncólogas expertas). Con todo ese material, realizamos, en primer lugar, un análisis cualitativo de dichas entrevistas para llegar a conocer las vivencias, las necesidades y posibles recursos para los adolescentes. En segundo lugar, cotejamos esta información con la recogida a partir de la literatura y, finalmente, elaboramos un listado de elementos que la página web debería contemplar.

\subsection{Análisis de las entrevistas}

En cuanto al análisis de las tres entrevistas con los hijos adolescentes, estos destacan que respecto a la información, agradecen que sean informados por el padre o la madre enfermo/a. Ante el cáncer parental presentan una amplia gama de reacciones. Remarcan reacciones emocionales como tristeza, rabia y culpa, pero también hablan 
de la impotencia y de la soledad. Suelen apoyar al padre o la madre enfermo/a y asimilan más responsabilidades en casa. El humor es una herramienta potente que les ayuda a gestionar mejor la situación. A los adolescentes les ayuda el apoyo de sus amigos, como también recibir más información por parte de los médicos. El poder implicarse en el proceso, como por ejemplo acompañar el padre o la madre a la quimioterapia, se vive positivamente. En una página web los adolescentes esperan encontrar información básica sobre la enfermedad, acceder a testimonios de otros adolescentes que han pasado por la misma situación, poder interactuar con estos adolescentes, y conocer estrategias para afrontar mejor la situación. El formato de la página web tiene que ser atractivo y llamativo.

En cuanto al análisis de las entrevistas con los padres, la mayoría de estos padres indican que han informado a sus hijos en el momento del diagnóstico con información clara y precisa. Admiten, no obstante, que la enfermedad es un tema tabú en casa. Pero en las entrevistas también hablamos con padres que se sienten incapaces de comunicar el diagnóstico al hijo/a y cuyos hijos se enteran de manera indirecta de la enfermedad. Según los padres, algunos hijos presentan reacciones emocionales como tristeza, pero a la vez suelen apoyar emocionalmente al padre o a la madre enfermo/a y ayudan más en casa. Sin embargo, otros hijos no muestran ninguna reacción emocional delante de los padres y no se implican en el proceso de la enfermedad. También se puede reducir su rendimiento académico. Respecto a las necesidades de los hijos, mencionan que es importante que tengan información sobre el cáncer y que han observado que buscan información en internet. Los hijos se sienten mejor cuando se pueden implicar en el proceso. Asimismo opinan que a sus hijos les ayudaría poder hablar con un psicólogo o también con adolescentes que están pasando por la misma situación.

Las psicooncólogas destacan que es de fundamental importancia que una persona cercana revele el diagnóstico del cáncer parental al hijo/a, proporcionándole la información poco a poco y respetándole si no quiere saber más. Según ellas los hijos pueden presentar reacciones emocionales como por ejemplo tristeza, rabia, apatía y preocupación. Pueden llegar a cambiar los roles con sus padres, sobreprotegiendo a estos y asimilando muchas más responsabilidades que antes en casa. Pero también hay hijos que aparentemente no les importa la enfermedad parental y no lo exteriorizan. Las psicooncólogas consideran que es fundamental que los hijos sigan su rutina diaria. En esta situación a los hijos les ayuda el apoyo de sus amigos, como también poder hablar con adolescentes que han pasado o están pasando por la misma experiencia. Es importante que el/la hijo/a se pueda implicar en el proceso de la enfermedad. Respecto a la página web subrayan la importancia de un formato actualizado y atractivo. Como contenido tiene que incluir información sobre la enfermedad y proporcionar diferentes estrategias de afrontamiento. Una página web tiene que ayudar a los hijos a normalizar la experiencia del cáncer parental. Asimismo puede tener la función de facilitar posteriores encuentros en persona con profesionales que les ayudan a manejar el cáncer parental.

\subsection{Comparación entre entrevistas y literatura e implicaciones para la página web}

Las Tablas 1, 2 y 3 muestran los acuerdos y desacuerdos entre la información obtenida en las entrevistas y la aportada por la literatura, estructurado en tres bloques: las reacciones de los adolescentes, sus necesidades, y sus recursos. A partir del acuerdo o desacuerdo entre las entrevistas y la literatura se realiza una propuesta concreta para la página web. 
Tabla 1. Acuerdos y desacuerdos entre la literatura y las entrevistas respecto a las reacciones por parte de los hijos y decisiones para la página web

\begin{tabular}{|c|c|c|}
\hline $\begin{array}{l}\text { Reacciones por } \\
\text { parte de los hijos }\end{array}$ & $\begin{array}{l}\text { Acuerdo/desacuerdo entre } \\
\text { literatura y entrevistas }\end{array}$ & $\begin{array}{l}\text { Conclusiones y decisiones } \\
\text { para la página web }\end{array}$ \\
\hline $\begin{array}{l}\text { Rendimiento } \\
\text { académico }\end{array}$ & $\begin{array}{l}\text { Acuerdo: Una posible } \\
\text { consecuencia al cáncer } \\
\text { parental puede ser una bajada } \\
\text { del rendimiento académico } \\
\text { del adolescente. }\end{array}$ & $\begin{array}{l}\text { Pensamos que un peor } \\
\text { rendimiento académico } \\
\text { es una reacción a todo lo } \\
\text { que implica el proceso de } \\
\text { la enfermedad parental. } \\
\text { Al tratarse de una } \\
\text { consecuencia del cáncer } \\
\text { parental, en la página web } \\
\text { no se trata directamente, } \\
\text { pues creemos que } \\
\text { trabajando los otros } \\
\text { aspectos afectados, el } \\
\text { rendimiento académico } \\
\text { también debería mejorar. }\end{array}$ \\
\hline Cambio de rol & $\begin{array}{l}\text { Acuerdo: Los adolescentes } \\
\text { pueden asumir más } \\
\text { responsabilidades en casa e } \\
\text { intercambiar los roles con sus } \\
\text { padres, lo cual puede llevar al } \\
\text { aislamiento y a la interrupción } \\
\text { de la vida social. }\end{array}$ & $\begin{array}{l}\text { Consideramos que asumir } \\
\text { más responsabilidad en } \\
\text { casa es una consecuencia } \\
\text { normal de la enfermedad } \\
\text { parental, pero el/la } \\
\text { adolescente tiene que } \\
\text { poder mantener al máximo } \\
\text { posible su rutina diaria y } \\
\text { seguir con sus actividades } \\
\text { lúdicas. En la página web } \\
\text { decidimos trabajar este } \\
\text { aspecto mediante pautas. }\end{array}$ \\
\hline $\begin{array}{l}\text { Impacto de los } \\
\text { cambios físicos en } \\
\text { el padre/madre }\end{array}$ & $\begin{array}{l}\text { Acuerdo: A los adolescentes } \\
\text { les provoca ansiedad ver los } \\
\text { cambios físicos y la debilidad } \\
\text { física en el padre o en la } \\
\text { madre enfermo y les cuesta } \\
\text { aceptarlo. }\end{array}$ & $\begin{array}{l}\text { Consideramos } \\
\text { importante trabajar el } \\
\text { impacto que produce } \\
\text { ver el padre o la madre } \\
\text { débil, para poder bajar } \\
\text { los niveles de ansiedad y } \\
\text { fomentar la aceptación. }\end{array}$ \\
\hline $\begin{array}{l}\text { Expresividad } \\
\text { emocional }\end{array}$ & $\begin{array}{l}\text { Acuerdo: En general los hijos } \\
\text { se muestran preocupados, } \\
\text { pero algunos también pueden } \\
\text { no mostrar ninguna reacción } \\
\text { emocional y no implicarse en } \\
\text { el proceso. Los padres pueden } \\
\text { creer que a sus hijos realmente } \\
\text { no les afecta su enfermedad y } \\
\text { pueden terminar subestimando } \\
\text { el impacto del diagnóstico } \\
\text { para los hijos. }\end{array}$ & $\begin{array}{l}\text { La página web ha de dejar } \\
\text { clara la importancia de } \\
\text { crear y proporcionar } \\
\text { recursos fuera del } \\
\text { entorno familiar que } \\
\text { ayuden a los adolescentes } \\
\text { a expresar la reacción } \\
\text { emocional ante el cáncer } \\
\text { parental. }\end{array}$ \\
\hline
\end{tabular}


Tabla 2. Acuerdos y desacuerdos entre la literatura y las entrevistas respecto a las necesidades de los hijos y decisiones para la página web

\begin{tabular}{|c|c|c|}
\hline Necesidades & $\begin{array}{l}\text { Acuerdo/ } \\
\text { desacuerdo entre } \\
\text { literatura y } \\
\text { entrevistas }\end{array}$ & $\begin{array}{l}\text { Conclusiones y decisiones para la } \\
\text { página web }\end{array}$ \\
\hline $\begin{array}{l}\text { Recibir } \\
\text { información }\end{array}$ & $\begin{array}{l}\text { Acuerdo: Los } \\
\text { adolescentes } \\
\text { manifiestan la } \\
\text { necesidad de recibir } \\
\text { más información } \\
\text { sobre el cáncer. }\end{array}$ & $\begin{array}{l}\text { Los adolescentes saben manejar } \\
\text { mejor la situación cuando reciben } \\
\text { más información. Por lo tanto la } \\
\text { página web tiene que incluir un } \\
\text { apartado con información. }\end{array}$ \\
\hline $\begin{array}{l}\text { Hablar con } \\
\text { adolescentes en la } \\
\text { misma situación }\end{array}$ & $\begin{array}{l}\text { Acuerdo: A los } \\
\text { adolescentes } \\
\text { les ayuda poder } \\
\text { hablar con otros } \\
\text { adolescentes que } \\
\text { han pasado o están } \\
\text { pasando por la } \\
\text { misma experiencia. }\end{array}$ & $\begin{array}{l}\text { Los adolescentes pueden pensar } \\
\text { que alguien que no ha pasado por la } \\
\text { misma situación que ellos no puede } \\
\text { entenderlos. Por lo tanto puede } \\
\text { ser útil hablar con adolescentes } \\
\text { que han pasado por lo mismo. } \\
\text { Se ofrece la posibilidad de } \\
\text { interacción en el foro de la página } \\
\text { web. }\end{array}$ \\
\hline $\begin{array}{l}\text { Implicarse en el } \\
\text { proceso }\end{array}$ & $\begin{array}{l}\text { Desacuerdo: En } \\
\text { la literatura hemos } \\
\text { encontrado muy } \\
\text { poca referencia a la } \\
\text { importancia de que } \\
\text { el/la adolescente } \\
\text { se puede implicar } \\
\text { en el proceso de } \\
\text { la enfermedad. } \\
\text { En las entrevistas } \\
\text { esta implicación } \\
\text { se ha comentado } \\
\text { ampliamente. }\end{array}$ & $\begin{array}{l}\text { La literatura que hemos consultado } \\
\text { mayoritariamente ha sido } \\
\text { anglosajona. Consideramos que } \\
\text { la implicación en el proceso } \\
\text { puede tener más importancia en } \\
\text { nuestro entorno cultural que en la } \\
\text { cultura anglosajona. Por lo tanto lo } \\
\text { consideramos importante trabajar y } \\
\text { decidimos incluir este tema en la } \\
\text { página web. }\end{array}$ \\
\hline $\begin{array}{l}\text { Comunicación } \\
\text { familiar }\end{array}$ & $\begin{array}{l}\text { Acuerdo: La } \\
\text { comunicación } \\
\text { familiar juega un } \\
\text { papel importante en } \\
\text { la adaptación a la } \\
\text { situación por parte } \\
\text { de los adolescentes. } \\
\text { Puede bajar el riesgo } \\
\text { de sufrir ansiedad y } \\
\text { depresión. }\end{array}$ & $\begin{array}{l}\text { En la literatura la importancia } \\
\text { de la comunicación familiar está } \\
\text { ampliamente documentada y en las } \\
\text { entrevistas también se comentó. } \\
\text { Sin duda es un factor a tener } \\
\text { en cuenta. En la página web no } \\
\text { podemos tener una influencia directa } \\
\text { sobre ello, pero consideramos } \\
\text { importante ofrecer pautas para } \\
\text { dar la posibilidad de que la } \\
\text { comunicación mejore. }\end{array}$ \\
\hline
\end{tabular}


Tabla 3. Acuerdos y desacuerdos entre la literatura y las entrevistas respecto a los posibles recursos y decisiones para la página web.

\begin{tabular}{|c|c|c|}
\hline Recursos & $\begin{array}{l}\text { Acuerdo/ } \\
\text { desacuerdo entre } \\
\text { literatura y } \\
\text { entrevistas }\end{array}$ & $\begin{array}{l}\text { Conclusiones y decisiones para la } \\
\text { página web }\end{array}$ \\
\hline Apoyo social & $\begin{array}{l}\text { Acuerdo: Los } \\
\text { adolescentes } \\
\text { perciben que en } \\
\text { general el entorno } \\
\text { les apoya poco. }\end{array}$ & $\begin{array}{l}\text { El hecho de que los adolescentes se } \\
\text { sientan poco apoyados, indica que } \\
\text { es necesario proporcionarles más } \\
\text { apoyo. En la página web se pretende } \\
\text { hacerles llegar este apoyo mediante } \\
\text { la comunidad de adolescentes que } \\
\text { están en la misma situación que ellos } \\
\text { y, también mediante los profesionales } \\
\text { que están a su disposición. }\end{array}$ \\
\hline $\begin{array}{l}\text { Estrategias de } \\
\text { afrontamiento } \\
\text { y reacciones } \\
\text { psicológicas }\end{array}$ & $\begin{array}{l}\text { Acuerdo: Para los } \\
\text { adolescentes puede } \\
\text { ser beneficioso } \\
\text { conocer y aprender } \\
\text { estrategias de } \\
\text { afrontamiento, } \\
\text { como también } \\
\text { saber cuáles son } \\
\text { las reacciones } \\
\text { psicológicas } \\
\text { habituales en esta } \\
\text { situación. }\end{array}$ & $\begin{array}{l}\text { Consideramos importante proporcionar } \\
\text { a los adolescentes la posibilidad } \\
\text { de aprender las estrategias de } \\
\text { afrontamiento que mayor eficacia han } \\
\text { demostrado. Se decide incluirlas en la } \\
\text { página web. }\end{array}$ \\
\hline $\begin{array}{l}\text { Expresión } \\
\text { artística }\end{array}$ & $\begin{array}{l}\text { Desacuerdo: En } \\
\text { las entrevistas } \\
\text { se comenta la } \\
\text { importancia de que } \\
\text { la página web sea } \\
\text { un lugar, dónde } \\
\text { los adolescentes } \\
\text { pueden dejar de } \\
\text { forma creativa } \\
\text { su testimonio y } \\
\text { también leer el } \\
\text { testimonio de otros. } \\
\text { En la literatura no } \\
\text { hemos encontrado } \\
\text { información concreta } \\
\text { sobre este tema. }\end{array}$ & $\begin{array}{l}\text { Consideramos importante que la } \\
\text { página web sea un lugar interactivo } \\
\text { y que los adolescentes también } \\
\text { pueden ayudar a crearla. Por lo tanto } \\
\text { decidimos incluir un apartado donde } \\
\text { pueden dejar de forma creativa su } \\
\text { testimonio o leer el testimonio de } \\
\text { otros adolescentes. }\end{array}$ \\
\hline
\end{tabular}




\begin{tabular}{|c|c|c|}
\hline Recursos & $\begin{array}{l}\text { Acuerdo/ } \\
\text { desacuerdo entre } \\
\text { literatura y } \\
\text { entrevistas }\end{array}$ & $\begin{array}{l}\text { Conclusiones y decisiones para la } \\
\text { página web }\end{array}$ \\
\hline $\begin{array}{l}\text { Importancia del } \\
\text { humor (sentirse } \\
\text { bien y hacer } \\
\text { sentir bien al } \\
\text { padre) }\end{array}$ & $\begin{array}{l}\text { Desacuerdo: } \\
\text { Utilizar el humor } \\
\text { como herramienta } \\
\text { para afrontar la } \\
\text { situación únicamente } \\
\text { se nombró en las } \\
\text { entrevistas con los } \\
\text { hijos. }\end{array}$ & $\begin{array}{l}\text { En la literatura no hemos encontrado } \\
\text { evidencia que demuestra que usar } \\
\text { el humor ayuda a sobrellevar mejor } \\
\text { la situación. Consideramos que } \\
\text { puede ser porque la mayoría de las } \\
\text { investigaciones realizadas son de otro } \\
\text { entorno cultural. Teniendo en cuenta } \\
\text { el factor cultural, vemos importante } \\
\text { incluir el elemento de humor en la } \\
\text { página web. Pero hace falta estudiar } \\
\text { más detalladamente su inclusión para } \\
\text { que sea beneficiosa. Por lo tanto se } \\
\text { propone incluir el humor en una futura } \\
\text { versión de la página web. }\end{array}$ \\
\hline Formato & $\begin{array}{l}\text { Desacuerdo: A } \\
\text { pesar de que en } \\
\text { la literatura no } \\
\text { hablan directamente } \\
\text { del formato de la } \\
\text { página web, en } \\
\text { las entrevistas sí } \\
\text { que remarcan la } \\
\text { importancia de un } \\
\text { formato atractivo y } \\
\text { llamativo. }\end{array}$ & $\begin{array}{l}\text { Cuando se tenga que realizar y crear la } \\
\text { página web, se tendrá que trabajar } \\
\text { muy detalladamente el diseño del } \\
\text { formato. }\end{array}$ \\
\hline
\end{tabular}

Tal y como se puede apreciar en la tabla 1, los adolescentes tienen una amplia gama de reacciones, cuyos efectos negativos se pretenden disminuir con la página web. En general los hijos adolescentes perciben que el entorno presta poca atención a lo que ellos necesitan y tampoco se sienten apoyados. En la tabla 2 se puede ver lo que ellos necesitarían en esta situación y como la página web intentará cubrir estas necesidades. Por último, y respecto a los recursos que ayudan a los adolescentes a llevar mejor la situación, tal y como se puede apreciar en la tabla 3, hay varios elementos cuya inclusión en la página web ya recomendamos, pero también hay elementos que será necesario estudiar más detalladamente para decidir cómo se incluirán en la página web.

Esta propuesta de elementos para la página web no está diseñada para hijos adolescentes cuyos padres han entrado en la fase paliativa, por lo que se debe seguir estudiando esta temática para discutir su inclusión en una futura versión de la página web. 


\section{Propuesta para la elaboración de los elementos de la página web}

A continuación pasamos a detallar la propuesta para la elaboración de los elementos de la página web (Ver Figura 1). Consideramos muy importante tener en cuenta en la página web el posible riesgo de iatrogenia. Por eso proponemos crear un sistema de filtros mediante unas preguntas que el/la adolescente tiene que contestar antes de poder acceder a los diferentes apartados de la página web. En función de estas respuestas, la página dirigirá al adolescente hacia los contenidos que sean adecuados para su situación intentando así evitar el riesgo de producir confusión o malestar.

Con esos requisitos, el diseño de la página web y de sus contenidos sería el que detallamos a continuación:

Figura 1. Visión global de los elementos de la página web

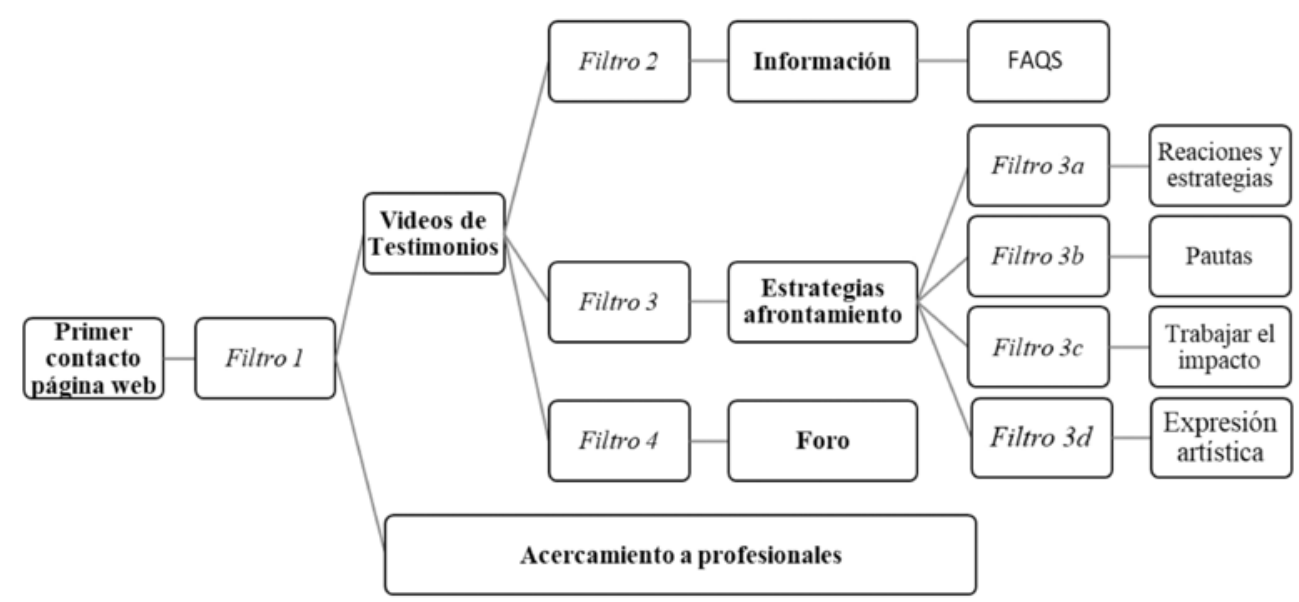

\section{Videos de testimonios}

Cuando el/la adolescente realiza el primer contacto con la página web, se aplica un primer filtro (Filtro 1) con preguntas para lograr hacer un primer perfil del adolescente. A partir de allí, se le ofrece ver un video corto con un testimonio de alguien que coincide con su edad y su género, y también con la gravedad y la fase de la enfermedad parental. Estos videos estarán diseñados expresamente para la página web y serán interpretados por actores. Con este video se pretende lograr que el/la adolescente se sienta identificado/a con el actor del video y por lo tanto que gane confianza y motivación para seguir utilizando la página web como herramienta en el afrontamiento del cáncer parental.

Después de ver el video, mediante otros filtros (Filtros 2, 3 y 4), se pretende llegar a establecer la combinación que mejor pueda adecuarse al adolescente integrando el recibir más información sobre el cáncer, el conocer estrategias de afrontamiento y la posibilidad de interactuar con otros adolescentes. 


\section{Información sobre el cáncer}

El adolescente que se puede beneficiar de recibir más información tiene acceso al apartado de la información. Esta información estará estructurada en diversos formatos a los que accederá el adolescente de acuerdo al perfil que surja de la aplicación del Filtro 2. En este apartado también se le ofrece la posibilidad de consultar más información mediante una lista de preguntas frecuentes (FAQS). Esta lista será adaptada al perfil del adolescente que surja de la combinación de los Filtros 1 y 2.

\section{Estrategias de afrontamiento}

Los filtros del apartado de estrategias de afrontamiento (Filtros 3a, 3b, 3c y 3d) valorarán el grado en que el adolescente necesita conocer recursos para afrontar mejor la situación. Según como contesta a las preguntas sobre si le gustaría conocerlos, se le redirige (o no) a cada uno de los subtemas para asegurar que el contenido de la página web realmente le ayuda. Se le propone conocer cuáles son las reacciones habituales que los adolescentes en la misma situación suelen tener, y también se le ofrece trabajar estrategias de afrontamiento que han demostrado su eficacia. Además, en este apartado se le ofrece la posibilidad de acceder a pautas para mantener la rutina diaria, para promover la comunicación familiar y también se trabaja cómo el/la adolescente se puede implicar más en el proceso. Asimismo, se ofrece la posibilidad de trabajar el impacto que los cambios físicos del padre o de la madre pueden tener en el/la adolescente.

Todos estos puntos nombrados en el apartado de estrategias de afrontamiento se intentarán completar mediante un apartado de expresión artística en el cual el/ la adolescente puede aportar su testimonio de forma libre, realizando por ejemplo un escrito o un poema, etc. También puede hacer uso de los modelos que la página web le propone para expresarse. El profesional filtrará su aportación y lo ubicará en la zona de la página web que pueda servir para otros adolescentes que se puedan beneficiar del contacto con ese tipo de expresión.

\section{Foro}

Para tener acceso al foro, el/la adolescente tiene que mostrar interés en querer hablar con otros adolescentes que están en la misma situación, lo cual se determina a través del Filtro 4. Este foro siempre tiene que estar bajo la supervisión de un profesional que intervendrá cuando sea necesario para facilitar la eficacia del funcionamiento del foro y evitar la iatrogenia.

\section{Acercamiento a profesionales}

Este apartado está abierto para todos los adolescentes después de haber contestado las preguntas del Filtro 1, no siendo necesario pasar por ningún filtro más. Se ofrece a los adolescentes la posibilidad de interactuar con profesionales por medio de un chat. Si lo desean pueden concretar una cita por Skype. Las respuestas del Filtro 1 ayudarán al profesional a tener una información inicial del adolescente. Es importante que los profesionales que ofrecen este servicio tengan disponibilidad y horarios concretos durante los cuales atiendan a los adolescentes, asegurando que una vez que el/la 
adolescente haya hecho la solicitud de contacto no tenga que esperar mucho tiempo hasta que se le atiende.

\section{Limitaciones}

Hay que tener en cuenta que la metodología utilizada en este trabajo para elaborar el listado de elementos de la página web tiene ciertas limitaciones, como por ejemplo el número reducido de las entrevistas realizadas. Así, solo hemos podido hablar con una paciente que está en fase terminal, madre de un hijo adolescente y con una hija cuyo padre también está en fase terminal, lo cual nos impide en estos momentos plantear cómo estructurar la página web para abordar adecuadamente la etapa terminal de padres o madres de los hijos adolescentes. Tampoco hemos podido determinar cómo podría utilizarse el humor.

Por otra parte, este trabajo es un primer boceto que debe proseguir y debe desarrollar aún más los elementos que lo constituyen, especialmente en lo referente a los filtros, ya que estos son la clave para que la página web sea útil y reduzca al máximo el riesgo de iatrogenia. Finalmente, hay que identificar los criterios estéticos y de formato que debe cumplir la página web para ser atractiva.

\section{Conclusiones}

Este trabajo ofrece un primer diseño de elementos para una página web. Estamos convencidos de que con una página web se ofrece una herramienta útil en el manejo del cáncer parental, pero es necesario seguir trabajando sobre el diseño y los diferentes apartados de la página web antes de poder llegar a realizarla. Consideramos que la creación de los filtros con las preguntas que los adolescentes deben contestar previamente al acceso a los contenidos, es una aportación importante para garantizar que los adolescentes solo llegan a los contenidos que les benefician. En este sentido, uno de los objetivos más importantes que perseguimos con la página web es disminuir la sensación de escaso apoyo que tiene el/la adolescente por parte del entorno, ofreciendo una comunidad de adolescentes que están en la misma situación que ellos y con los cuales pueden interactuar en el foro, así como también con profesionales a su alcance. También es importante que el adolescente reciba una información adecuada y una orientación respecto a las estrategias de afrontamiento que puede utilizar.

\section{Referencias bibliográficas}

1. Galceran J, Ameijide A, Carulla M, Mateos A, Quirós J, Rojas D, et al. Cancer incidence in Spain, 2015. Clin Transl Oncol 2017;19:799-825. doi: 10.1007/s12094-016-1607-9

2. Morris JN, Martini A, Preen D. The well-being of children impacted by a parent with cancer: an integrative review. Support Care Cancer 2016;24:3235-51. doi: 10.1007/ s00520-016-3214-2 
3. Forrest G, Plumb C, Ziebland S, Stein A. Breast cancer in the family-children's perceptions of their mother's cancer and its initial treatment: qualitative study. BMJ. 2006; doi:10.1136/bmj.38793.567801.AE

4. Huang X, O'Connor M, Lee S. School-aged and adolescent children's experience when a parent has non-terminal cancer: a systematic review and meta-synthesis of qualitative studies. Psychooncology 2014;23:493-506. doi: 10.1002/pon.3457

5. Giesbers J, Verdonck-de Leeuw I, Van Zuuren F, Kleverlaan N, Van der Linden M. Coping with parental cancer: web-based peer support in children. Psychooncology 2010;19:88792. doi: 10.1002/pon.1636

6. Grabiak B, Bender C, Puskar K. The impact of parental cancer on the adolescent: an analysis of the literature. Psychooncology 2007;16:127-37. doi: 10.1002/pon.1083

7. Küçükoğlu S, Çelebioğlu A. Identification of psychological symptoms and associated factors in adolescents who have a parent with cancer in Turkey. Eur J Oncol Nurs 2013;17:75-80. doi:10.1016/j.ejon.2011.10.008

8. Visser A, Huizinga G, Van der Graaf W, Hoekstra H, Hoekstra-Weebers J. The impact of parental cancer on children and the family: A review of the literature. Elsevier 2004;30:683-94.

9. Niemelä M, Hakko H, Räsänen S. A systematic narrative review of the studies on structured child-centred interventions for families with a parent with cancer. Psychooncology 2010;19:451-61. doi: 10.1002/pon. 1620

10. Krattenmacher T, Kühne F, Führer D, Beierlein V, Brähler E, Resch F, et al. Coping skills and mental health status in adolescents when a parent has cancer: A multicenter and multi-perspective study. J Psychosom Res 2013;74:252-9. doi: 10.1016/j. jpsychores.2012.10.003

11. Fernández Sánchez B, Jorge Martin V, Sánchez Vázquez C, Bejar Alonso E. Atención psicológica para pacientes con cáncer y sus familiares: ¿qué nos encontramos en la práctica clínica? Psicooncología 2016;13:191-204. doi: 10.5209/PSIC.54432

12. Sears HA, Sheppard HM. I just wanted to be the kid: adolescents girls' experiences of having a parent with cancer. Can Oncol Nurs J 2004;14:18-20.

13. Tucker A, Sugerman D, Zelov R. On belay: Providing connection, support, and empowement to children who have a parent with cancer. J Experiential Educ 2013;36:93105. doi: 10.1177/1053825913487889

14. Rodríguez Morera A. ¿Cómo explican los niños y adolescentes el cáncer avanzado o terminal de sus padres?. Psicooncología 2015;12:171-82. doi: 10.5209/rev_PSIC.2015. v12.n1.48911

15. Fuentes S. Comunicació en cáncer: análisis de les preocupacions i dels recursos dels pares recent diagnosticats al comunicar la malatia als seus fills. [tesis doctoral]: Universtiat Autònoma de Barcelona; 2015. Recuperada de Teseo.

16. Osborn T. The psychosocial impact of parental cancer on children and adolescents: a systematic review. Psychooncology 2007;16:101-26. doi: 10.1002/pon.1113

17. Phillips F. Adolescents living with a parent with advanced cancer: a review of the literature. Psychooncology. 2014;23:1323-39. doi: 10.1002/pon.3570

18. Berger K. Psicología del desarrollo: Infancia y Adolescencia. $9^{a}$ ed. Madrid: Panamericana, 2016.

19. World Health Organization. Health for the world's adolescents: A second chance in the second decade [Internet]. WHO 2014. [acceso 15 de enero de 2019]. Disponible en: e: http://apps.who.int/adolescent/second-decade 
20. Morris JN, Ohan J, Martini A. An investigation of support services available by Internet searching to families impacted by a parents cancer. Psychooncology 2018:27:114-124. doi: 10.1002/pon.4478

21. Inhestern L, Haller A, Wlodarczyk O, Bergelt C. Psychosocial Interventions for families with parental cancer and barriers and facilitators to implementation and use - a systematic review. PLOS ONE. 2016;11(6):1-20. doi:10.1371/journal.pone.0156967.

22. Rutten L, Squiers L, Treiman K. Requests for information by family and friends of cancer patients calling the National Cancer Institute's Cancer Information Service. Psychooncology 2006;15:664-72. doi: 10.1002/pon.995 